Meta

Journal des traducteurs

Translators' Journal

\title{
La traducción del discurso oral de la literatura infantil y juvenil alemana: las partículas modales ja y eben/halt al euskera
}

\section{Naroa Zubillaga Gómez}

Volume 60, numéro 3, décembre 2015

URI : https://id.erudit.org/iderudit/1036146ar

DOI : https://doi.org/10.7202/1036146ar

Aller au sommaire du numéro

Éditeur(s)

Les Presses de l’Université de Montréal

ISSN

0026-0452 (imprimé)

1492-1421 (numérique)

Découvrir la revue

Citer cet article

Zubillaga Gómez, N. (2015). La traducción del discurso oral de la literatura infantil y juvenil alemana: las partículas modales $j a$ y eben/halt al euskera. Meta, 60(3), 621-637. https://doi.org/10.7202/1036146ar

\section{Résumé de l'article}

Le but de cet article est de résumer les résultats de l'analyse de la traduction de certaines particules modales allemandes en basque. Les données proviennent de notre thèse sur la traduction de la littérature allemande pour enfants en langue basque, qui a été menée selon une méthodologie descriptive et avec un vaste corpus numérique de 33 oeuvres originales allemandes et leurs traductions. Étant donné que le corpus renferme à la fois des traductions directes et indirectes, cet article examine également les différents résultats obtenus en comparant les traductions directes et indirectes. Les particules modales allemandes sont très fréquentes, surtout dans le discours oral et dans le discours écrit informel. Ainsi, l'analyse de la traduction de ces particules dans une langue minoritaire comme le basque nous permet de plonger dans le problème de la traduction des éléments discursifs oraux dans une langue où la création de l'oralité fictive semble assez complexe. Une attention toute particulière est donnée à la mesure du niveau de standardisation et à l'observation des cas d'interférence. 


\title{
La traducción del discurso oral de la literatura infantil y juvenil alemana: las partículas modales ja y eben/halt al euskera
}

\author{
NAROA ZUBILLAGA GÓMEZ \\ Universidad del País Vasco/Euskal Herriko Unibertsitatea, Vitoria-Gasteiz, España \\ naroa.zubillaga@ehu.es
}

\begin{abstract}
RÉSUMÉ
Le but de cet article est de résumer les résultats de l'analyse de la traduction de certaines particules modales allemandes en basque. Les données proviennent de notre thèse sur la traduction de la littérature allemande pour enfants en langue basque, qui a été menée selon une méthodologie descriptive et avec un vaste corpus numérique de 33 œuvres originales allemandes et leurs traductions. Étant donné que le corpus renferme à la fois des traductions directes et indirectes, cet article examine également les différents résultats obtenus en comparant les traductions directes et indirectes. Les particules modales allemandes sont très fréquentes, surtout dans le discours oral et dans le discours écrit informel. Ainsi, l'analyse de la traduction de ces particules dans une langue minoritaire comme le basque nous permet de plonger dans le problème de la traduction des éléments discursifs oraux dans une langue où la création de l'oralité fictive semble assez complexe. Une attention toute particulière est donnée à la mesure du niveau de standardisation et à l'observation des cas d'interférence.
\end{abstract}

\section{ABSTRACT}

The goal of this article is to sum up the results of the analysis of the translations of some German modal particles into Basque. The data derives from our thesis on the translation of German children's literature into Basque, which was conducted following a descriptive methodology and using a wide digital corpus of 33 original German works and their translations. As in the corpus there are direct as well as indirect translations, this article also examines the different results obtained by comparing direct and indirect translations. German modal particles are very frequent, especially in oral and informal written speech. Thus, the analysis of their translation into a minority language like Basque enables us to delve into the problem of translating oral discursive elements into a language where the creation of fictional orality seems to be fairly complex. A special attention is given to measuring the level of standardization and observation of interference cases.

\section{MOTS-CLÉS/KEYWORDS}

particule modale, littérature pour enfants, traduction indirecte, standardisation, interférence modal particle, children's literature, indirect translation, standardization, interference

\section{Introducción}

El objetivo del presente artículo es presentar parte de los resultados obtenidos de la tesis doctoral Traducción de literatura infantil y juvenil al euskera: comparación de traducciones directas e indirectas a través de un corpus (Zubillaga 2013; nuestra traducción). En dicha tesis doctoral se analizó, en primer lugar, el catálogo de las obras de literatura infantil y juvenil (LIJ) alemanas traducidas a la lengua vasca (Zubillaga 
2009) así como los sistemas literarios correspondientes; después, tras la selección de algunas de las obras más representativas y su digitalización y alineación, se analizaron en el corpus compilado elementos lingüísticos más específicos como la traducción de los insultos y maldiciones y la traducción de algunas partículas modales alemanas. La finalidad de estos análisis concretos era la aproximación al estudio de la traducción al euskera del discurso oral de la LIJ. Somos conscientes de que el discurso oral lo componen diversas características lingüísticas y textuales, por lo que un estudio integral del mismo exigiría analizar los diferentes aspectos. Sin embargo, el presente artículo resume los resultados derivados del análisis de la traducción de algunas partículas alemanas, concretamente, de las partículas alemanas ja y eben/halt.

El estudio de la traducción de elementos discursivos orales, como lo son en este caso las partículas modales alemanas, resulta de gran interés en una lengua como la vasca. La estandarización de la lengua vasca es relativamente joven, ya que el euskera batua [vasco estándar] se creó en 1968. El régimen franquista finalizó en 1975, y en 1979 entró en vigor el decreto del bilingüismo, lo que provocó el aumento de las matriculaciones en las ikastolas [escuelas vascas]. La inclusión de la lengua vasca en el sistema educativo vasco provocó la necesidad de disponer de materiales en euskera, y el siguiente dato así lo demuestra: mientras que antes de los años 1980 se publicaban en euskera poco más de una docena de libros de LIJ al año, a partir de los años 1980 se empezaron a publicar más de 300 al año (Etxaniz 2011: 24). Tal demanda provocó el aumento de la práctica traductora. Sin embargo, desde el punto de vista lingüístico, el objetivo era asegurar la supervivencia y, por tanto, el aprendizaje de la lengua. En tales circunstancias, la LIJ cumple un papel fundamental, ya que se trata de un género ubicado entre el sistema educativo y el literario (Shavit 1986) y, por tanto, resulta clave en una situación de recuperación lingüística. Observamos el caso, por ejemplo, de la LIJ alemana traducida al hebreo en la época posterior a la segunda Guerra Mundial, donde los traductores introducen varios sinónimos de una misma palabra, tratando de utilizar un lenguaje elegante y evitando el registro oral (Ben-Ari 1992: 221-231). En el caso de la lengua vasca, durante años los esfuerzos también se dirigieron a establecer una lengua sobre todo formal y estándar, mientras que el uso más natural de los dialectos quedó en segundo plano (Uribarri 2011: 257-258). Esto afecta a la lengua utilizada en el género de la LIJ, sobre el que aún hoy cuenta-cuentos vascos como Añorga opinan que debería acercarse más al registro oral:

[...] el libro no está preparado que se lea en voz alta; o dicho de otra manera: el libro no tiene desarrollado su oralidad. Y, en mi opinión, si desarrollamos la oralidad, nos acercaremos más a los lectores más pequeños, y lograremos una mejor comunicación. ¿Por qué? Pues porque los niños viven más cerca de la comunicación oral que de la escrita. (Añorga 2011: 22; nuestra traducción)

Es bien sabido que el registro escrito nunca es fiel reflejo del registro oral, pero mediante la inserción de características orales tales como las repeticiones, las elipsis o las dudas, los discursos tanto narrativos, audiovisuales como de teatro intentan construir una oralidad fingida. En los últimos años ha aumentado el interés por el estudio contrastivo y la traducción de este tema, particularmente en el grupo de investigación de Barcelona TRADIF (la TRAducción del DIálogo Ficcional) por los trabajos publicados en este ámbito (Brumme y Espunya 2012; Fischer y Wirf Naro 2012). Hasta el momento, no se ha realizado ningún estudio sobre el modelo de lenguaje utilizado en la LIJ vasca. Como excepción, podríamos citar la tesis doctoral de 
Barambones (2010), quien analizó las traducciones del inglés al euskera de los productos audiovisuales de la programación infantil en la televisión vasca. En su estudio, Barambones concluyó que el lenguaje utilizado era demasiado formal y demasiado cercano al registro escrito (Barambones 2012: 165). El esfuerzo por reflejar por escrito el discurso oral de los más jóvenes resulta un tanto complicado en euskera, puesto que se trata de una lengua mixta, en efecto los jóvenes hacen uso de otras lenguas - sobre todo del español - para crear su propio discurso (Esnaola 1997: 31). El lenguaje juvenil es un lenguaje que tiene sus propias características pero que mantiene con el lenguaje formal una relación de enriquecimiento mutuo: el lenguaje juvenil deconstruye el lenguaje estándar (creando nuevos significados etc.), pero, al mismo tiempo, el lenguaje estándar acepta las nuevas palabras y expresiones creadas por el lenguaje juvenil (Neuland 2007: 144). En una lengua que no se halla en una situación diglósica se dan las condiciones necesarias para que surja el enriquecimiento mutuo entre el lenguaje juvenil y el lenguaje formal. Sin embargo, ello resulta bastante dudoso en la lengua vasca. En variedades dialectales existen expresiones de lenguaje juvenil que la lengua estándar ha aceptado con las correspondientes adaptaciones de grafía etc. pero en lo que se refiere a la creación de nuevas expresiones, los jóvenes vascos apenas deconstruyen el vasco estándar. Por tanto, hacen uso de recursos de otras lenguas y, por ello, la variedad estándar no refleja las palabras que los jóvenes utilizan todos los días. Así, la labor del traductor al intentar traducir al euskera la oralidad fingida de los más jóvenes se ve a veces obstaculizada por la dificultad de tener que decidir si opta por utilizar expresiones dialectales que tal vez no todos los lectores puedan conocer, si se decanta por expresiones que sean fieles al registro oral pero que pueden reflejar interferencias de otras lenguas mayoritarias o si escoge expresiones en lengua vasca estándar que neutralizan o disfemizan ${ }^{1}$ claramente el tono y el registro del texto original. La creación de la oralidad fingida resulta, por tanto, un tema más complejo en lenguas minorizadas ${ }^{2}$ como el euskera.

Las partículas modales alemanas (Abtönungspartikeln) son palabras que no se declinan y que no son ni adverbios, ni palabras modales, ni preposiciones, ni conjunciones (Helbig 1988: 20). Cumplen una función comunicativo-pragmática y definen la relación entre los interlocutores, es decir, informan sobre la función ilocutiva de la frase. Por todo ello, se encuentran sobre todo en registros no formales y en el discurso oral. En cuanto a la literatura, «se las encuentra sobre todo en textos que reflejan el registro coloquial, es decir, un registro que establece una cierta cercanía entre los interlocutores cuya expresión formal es normalmente un diálogo» (Prüfer 1995: 16). Es de esperar, pues, que en textos de LIJ nos encontremos con una cantidad considerable de partículas modales, y siendo éstas elementos del discurso oral, resulta de gran interés observar qué estrategia se utiliza para su traducción al euskera.

La traducción de las partículas modales ha suscitado siempre interés, y no faltan estudios sobre su traducción al español. Cabe señalar el trabajo de Waltereit (2006), quien analiza los equivalentes de las partículas modales alemanas en lenguas románicas en general. La traducción en concreto al español fue estudiada primero por Christiane Beerbom (1992), quien analizó la traducción de ja, doch, schon, eben y halt; más tarde por Prüfer (1995) quien analizó los equivalentes tanto al castellano como al inglés; Cárdenes (1997) analizó la traducción al español de las partículas aber, denn, doch y eben; y, por último, Ferrer (1999) analizó las equivalencias al 
español de las partículas modales en modo interrogativo. Hasta ahora, no se ha realizado ningún trabajo académico que analice posibles equivalencias en euskera, exceptuando la gramática sobre la lengua vasca en alemán redactada por Christiane Bendel (2006), en la que la autora propone las partículas en euskera al, bide, omen/ ei y ote como equivalentes a algunas de las partículas alemanas, pero se trata de un apartado breve dentro de una gramática, y no está sustentado por un análisis específico. Es, por tanto, un ámbito que está aún por estudiar. Por ello, en nuestro análisis hemos querido realizar un estudio empírico y observar las estrategias de traducción en euskera de algunas de las partículas modales.

Además de poner el acento en la traducción del discurso oral, el presente trabajo integra el análisis de la práctica traductora indirecta. El análisis de las traducciones de una lengua extranjera al euskera nos lleva a tener en cuenta una cuestión de considerable calado metodológico. Se trata de las traducciones indirectas, es decir, traducciones realizadas no directamente de la lengua original sino a partir de un texto y una lengua intermediarios. En el caso concreto de la traducción de LIJ alemana al euskera, la traducción indirecta ha sido una práctica bastante habitual (Zubillaga 2012: 83), y aunque en los últimos años haya aumentado el porcentaje de traducciones directas, un análisis descriptivo de la práctica traductora alemán/euskera exige tener en cuenta y analizar la traducción indirecta. El análisis que presentamos en este artículo distingue desde el principio traducciones supuestamente directas e indirectas, ya que uno de los principales objetivos es observar posibles diferencias en las estrategias de traducción correspondientes. Así pues, al introducir los datos de las obras que constituían el catálogo, además de la habitual información como el nombre del autor/ traductor, el año de publicación tanto del original como de la traducción etc. hemos incorporado el dato relativo al tipo de traducción: si se ha realizado directamente o indirectamente a partir de una versión intermediaria. Se trata de una información que normalmente las bases de datos bibliográficas no aportan, por lo que nos pusimos en contacto con las editoriales e incluso con algunos traductores para preguntarles este detalle que para nuestra investigación resultaba imprescindible.

Sin embargo, y aunque pudimos completar este dato en la mayoría de los casos, quisiéramos destacar el carácter provisional del mismo. La realización del catálogo supone el punto de partida de nuestra investigación; el posterior análisis textual podrá tal vez confirmar o desmentir el carácter de «supuesta traducción directa/ indirecta $»^{3}$ atribuido a las entradas del catálogo. Efectivamente, diversos estudios demuestran que resulta complicado decidir si una traducción es directa o indirecta (Ringmar 2007; Pięta 2012). La lengua vasca y su sistema cultural conviven con la lengua española y su sistema cultural en el Estado español y con la lengua francesa y su sistema cultural en el Estado francés; ello supone, entre otras consecuencias, que las publicaciones de las traducciones en español o francés sean normalmente previas a las publicaciones en euskera. La presencia de ambas lenguas mayoritarias en el territorio donde se habla la lengua vasca es, además, ineludible, por lo que analizar las posibles diferencias o no-diferencias entre las traducciones supuestamente directas e indirectas posibilita ahondar en la posición de la lengua vasca para con las lenguas española y francesa.

En este sentido, consideramos que el análisis de traducción entre una lengua hegemónica y una minorizada resulta especialmente interesante. Los estudios de traducción acostumbran a dirigir su atención a las relaciones entre dos o más lenguas 
hegemónicas. Sin embargo, precisamente son las minorías y las diferentes situaciones traductológicas en torno a ellas las que dejan ver nuevos horizontes: «Minorities bring inpredictable variations to constants and standards in the study of translating» (Venuti 1998: 143).

\section{Metodología}

Siguiendo los criterios utilizados en el seno del grupo de investigación consolidado TRALIMA/ITZULIK ${ }^{4}$ de la Universidad del País Vasco, el trabajo que sustenta esta aportación se realizó adoptando un marco metodológico acorde a los Estudios Descriptivos de Traducción (EDT) y siguiendo el esquema que Lambert y van Gorp (1985) propusieron para la realización de un estudio descriptivo: en primer lugar, compilamos un catálogo de las obras de LIJ alemana traducidas al euskera y describimos la situación de ambos sistemas literarios. Una vez analizado el catálogo, se establecieron los criterios para seleccionar los textos que constituirían el corpus. Por un lado, tuvimos en cuenta traducciones supuestamente directas e indirectas; tratamos de mantener una variedad equilibrada entre autores fuente, autores meta, editoriales y edad a la que estaban dirigidas las obras; seleccionamos traducciones realizadas a partir de 1980, ya que nuestra intención era hacer un estudio sincrónico sobre la realidad contemporánea de la traducción del alemán al euskera. Después, utilizamos dicho corpus para realizar el análisis macrotextual, el análisis de los paratextos, así como el análisis microtextual, es decir, el análisis de la traducción de los insultos y maldiciones y el análisis de la traducción de algunas partículas modales alemanas.

Se trata de un corpus digitalizado de 33 obras originales, 19 textos que supuestamente se tradujeron directamente y 14 textos que se tradujeron indirectamente. Con la ayuda de un informático colaborador del proyecto TRACE ${ }^{5}$, se dispuso de un programa que daba la opción de alinear hasta tres versiones. Se trata de un programa que etiqueta cada fichero en el nivel de la oración y, después, vincula o alinea los textos según se corresponden las etiquetas. El objetivo era tener digitalizadas y alineadas tanto las traducciones supuestamente directas (los textos en alemán y euskera) como las traducciones indirectas (los textos en alemán, en español y en euskera). Después subimos los textos alineados con sus traducciones al gestor de base de datos $M y S q l$ y enlazamos esta base de datos a un buscador creado también por el mismo colaborador informático. El proceso de creación del corpus ha sido laborioso (Zubillaga, Sanz et al. 2015), pero de este modo tenemos acceso a un corpus digital específico de traducciones de LIJ alemana al euskera de en total de $80 \operatorname{textos}^{6} \mathrm{y}$ 1.276.280 palabras, en el que podemos realizar búsquedas cómodamente, ya que introducimos en el buscador las palabras que nos interesan y al instante obtenemos todos los resultados de nuestro corpus que coinciden con la búsqueda.

En cuanto a la metodología utilizada para el análisis de la traducción de las partículas modales, el primer paso fue decidir qué partículas modales escoger. Con el fin de conocer la frecuencia de cada partícula en nuestro corpus, hemos utilizado el programa WordSmith para contabilizar la frecuencia de cada una de ellas. Teniendo en cuenta que las partículas modales alemanas tienen homónimos, hemos eliminado todas las partículas que aparecían en posición inicial, pues aquellas que cumplen la función de partícula modal alemana no aparecen en dicha posición. Así, después de 
eliminar las que estaban en posición inicial, contabilizamos la frecuencia de cada partícula. Asimismo, hemos tenido en cuenta que, según Prüfer (1995), las partículas modales más frecuentes son doch, denn y ja. Hemos querido analizar alguna de ellas y hemos elegido ja, pues en nuestro corpus es la que con más frecuencia aparece (1492 veces). Como era imposible analizar todos los ejemplos, seleccionamos los diez primeros casos de cada obra de nuestro corpus. Así, de las 33 obras que componen nuestro corpus extrajimos un total de 264 casos. Para la elección de otra partícula modal no quisimos utilizar el mismo criterio de la partícula más frecuente. Al contrario, quisimos elegir aquella que nos permitiese analizar todos los casos que nuestro corpus ofrecía. Por eso, optamos por las partículas sinónimas eben/halt (eben se utiliza más al norte de Alemania, y halt al sur). Hemos obtenido 222 resultados de eben y 62 de halt.

Los resultados que presentamos en este artículo se refieren, por tanto, a dos partículas modales. Un análisis completo exigiría un estudio integral de todas las partículas modales, pero este primer trabajo sirve como punto de partida y muestra del análisis de traducción del discurso oral. Tal y como hemos mencionado anteriormente, hemos tratado de realizar el estudio de traducción desde un punto de vista descriptivo, comparando las traducciones supuestamente directas con las traducciones indirectas. Al mismo tiempo, hemos querido observar si en nuestro corpus se cumplen las dos leyes propuestas por Toury (2012), la ley de la estandarización y la ley de la interferencia, y hemos querido hacer nuestra aportación a la formulación de dichas leyes.

Recordemos que la ley de la estandarización se refiere a que las traducciones tienden a utilizar un lenguaje más monótono: «in translation, items tend to be selected on a level which is lower than the one where textual relations have been established in the source text» (Toury 2012: 305; enfatizado en el original). En cuanto a la ley de la interferencia, Toury defiende que la lengua de partida siempre se ve reflejada en la lengua meta: "[...] phenomena pertaining to the make-up of the source text tend to force themselves on the translators and be transferred to the target text» (Toury 2012: 310).

Pensamos que concretamente la ley de la interferencia puede adquirir diferentes grados y matices según se trate de una traducción directa o indirecta. Cuando Toury formula la ley de la interferencia (Toury 2012: 310) lo hace refiriéndose a la interferencia causada por un texto original en su traducción. Sin embargo, ha de tenerse en cuenta que si se trata de una traducción indirecta, es decir, realizada en dos pasos, dicha interferencia puede resultar doble: la ocasionada por el texto original (derivada a través del texto puente) y la causada por la misma versión puente. En lo que se refiere a las traducciones directas realizadas a una lengua minorizada como el euskera, queremos observar si también existen indicios de existencia de una versión intermediaria, ya que en el caso de lenguas diglósicas, tal y como hemos comentado anteriormente, la presencia de la lengua mayoritaria es relativamente grande. Con lo cual, prestaremos especial atención a los indicios de interferencia en las supuestas traducciones directas. 


\section{Resultados}

A continuación resumiremos los principales resultados obtenidos en el análisis de las traducciones al euskera de las partículas modales ja y eben/halt. Para ello, primero explicaremos la función pragmática que cumple cada una de las partículas, el matiz semántico que aporta cada una de ellas. Después, observaremos las estrategias de traducción utilizadas para traducirlas al euskera, primero, teniendo en cuenta todos los resultados en conjunto, después, separándolos según hayan sido traducciones directas o indirectas.

\subsection{Resultados del análisis de la partícula ja}

Después de recoger todos los casos de la partícula ja en una tabla, pasamos a hacer una clasificación, pues no todas las partículas ja tenían el mismo matiz. Para ello nos basamos en la clasificación que propone Waltereit (2006: 39-44), según la cual, las funciones que la partícula ja cumple como partícula modal se dividen en los siguientes tres grupos: ja de conocimiento, donde el emisor tiene el convencimiento de que el receptor está al tanto de la información o incluso de que comparte la opinión (Er isst ja am liebsten Erdbeertorte [Pues es que / Como bien sabes, su comida favorita es la tarta de fresas]); ja de asombro, donde el emisor expresa el carácter evidente de la información, algo que el receptor no puede negar (Du hast ja ein Loch in Deinem Ärmel! [jPero si tienes un agujero en la manga!]); ja imperativo, donde el emisor expresa su deseo de que el receptor acepte lo que se le ordena (Komm ja nicht zu spät nach Hause! [jY que no vengas / Más te vale no venir demasiado tarde a casa!]. Tal y como confiesa el autor, la división de los tres grupos no siempre resulta tan sencilla como parece. Según nuestra clasificación, la mayoría de los ja son de conocimiento (191 en total), el segundo grupo es de los de asombro (50) y, por último, los de imperativo (12).

Tras analizar los casos y observar los resultados, destaca la estrategia de no traducir la partícula (84\%). Los casos en que se ha reflejado el matiz de la partícula son muy pocos $(16 \%)$ :

FIGURA 1

Porcentajes de las estrategias de traducción de la partícula ja

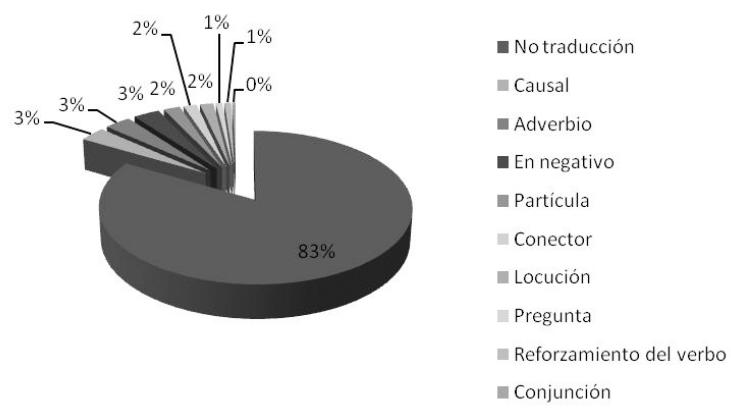

Quisiéramos destacar el carácter descriptivo de este análisis, por lo que la no-traducción de la partícula es considerada como estrategia de traducción, estrategia que además es la más utilizada. Estudios previos sobre el tema han distinguido entre traducción y no-traducción y se han centrado en las estrategias lingüísticas que 
dispone el español para la traducción de la partícula (Beerbom 1992; Prüfer 1995; Cárdenes 1997 o Ferrer, 1999); entendemos que de este modo se desaprueba la notraducción y, por tanto, se dispone de una actitud prescriptiva más que descriptiva. Sin embargo, la no-traducción es, a nuestro parecer, una estrategia de traducción como las demás. Además, siendo la no-traducción la estrategia más habitual, resulta aún más evidente tenerla en cuenta y tratar de analizar las razones para ello. Los factores causantes de la no-traducción de las partículas pueden ser varios: el tiempo limitado y la prisa a la hora de traducir, el conocimiento insuficiente de la lengua origen en el caso de las traducciones directas y la existencia de la práctica de la traducción indirecta. Sin embargo, también cabe la posibilidad de que los traductores vascos favorezcan en algunos casos la no-traducción de la partícula por considerarla la estrategia más adecuada.

A continuación comentaremos algunos de los ejemplos de las 10 estrategias utilizadas para la traducción de ja, empezando desde la no-traducción:

CUAdRO 1

Estrategias de traducción de la partícula ja

\begin{tabular}{|c|c|c|}
\hline Texto original & Versión puente & Texto meta \\
\hline $\begin{array}{l}\text { 1. „Ich bin ja ganz schwarz } \\
\text { geworden! [iPero si me he vuelto } \\
\text { completamente negro!] }\left(\mathrm{DKG}^{7}\right)\end{array}$ & $\begin{array}{l}\text {-Me he vuelto } \\
\text { completamente negro. } \\
\left(\mathrm{DKG}-\mathrm{es}^{8}\right)\end{array}$ & $\begin{array}{l}\text {-Erabat beltz bihurtu naiz. [Me he } \\
\text { vuelto completamente negro.] } \\
\left(\mathrm{DKG}-\mathrm{eu}^{9}\right)\end{array}$ \\
\hline $\begin{array}{l}\text { 2. „Das ist ja der Absatz von einem } \\
\text { Schuh!” [iPero si esto es el tacón } \\
\text { de un zapato!] }\left(\mathrm{DASH}^{10}\right)\end{array}$ & $\begin{array}{l}\text {-Pero ¡si esto es el tacón } \\
\text { de un zapato! } \\
\left(\mathrm{DASH}-\mathrm{es}^{11}\right)\end{array}$ & $\begin{array}{l}\text {-Zapata baten takoia da! [¡Es el } \\
\text { tacón de un zapato!] (DASH-eu }{ }^{12} \text { ) }\end{array}$ \\
\hline $\begin{array}{l}\text { 3. Aber, lieber Schwiegersohn, sage } \\
\text { mir doch, woher ist das viele Gold? } \\
\text { Das sind ja gewaltige Schätze! } \\
\text { [Pero, querido yerno, cuéntamelo, } \\
\text { ¿de dónde ha salido todo este oro? } \\
\text { ¡si es que se trata de una riqueza } \\
\text { inmensa!] }\left(\mathrm{KHM}^{13}\right)\end{array}$ & & $\begin{array}{l}\text { Baina, suhi estimatua, esaidak } \\
\text { nongoa den urre hori guztia, } \\
\text { egundoko altxorra duk eta! [Pero, } \\
\text { estimado yerno, dime de dónde } \\
\text { viene todo este oro, ya que es un } \\
\text { tremendo tesoro!] (KHM-eu }{ }^{14} \text { ) }\end{array}$ \\
\hline $\begin{array}{l}\text { 4. Steige ja nicht vorher aus, etwa } \\
\text { am Bahnhof Zoo oder auf einer } \\
\text { anderen Station!” ŁiQue no te } \\
\text { vayas a bajar antes, por ejemplo en } \\
\text { la estación del Zoo o en otra } \\
\text { cualquiera!] }\left(\mathrm{ED}^{15}\right)\end{array}$ & $\begin{array}{l}\text { ¿Que no te vayas a bajar } \\
\text { antes, por ejemplo en la } \\
\text { estación del Jardín } \\
\text { Zoológico o en otra } \\
\text { cualquiera! }\left(\mathrm{ED}-\mathrm{es}^{16}\right)\end{array}$ & $\begin{array}{l}\text { Etzaitezela lehenago jaitsi, } \\
\text { Zooparkeko geltokian esate } \\
\text { baterako, edo beste edozeinetan! } \\
\text { [iQue no te vayas a bajar antes, en } \\
\text { el parque del Zoo, por ejemplo, o } \\
\text { en cualquier otra!] (ED-eu }{ }^{17} \text { ) }\end{array}$ \\
\hline $\begin{array}{l}\text { 5. Sie will ja auch, daß sie Angst } \\
\text { haben und sich aufregen. [Y es que } \\
\text { eso es lo que quiere, que tengan } \\
\text { miedo que se pongan nerviosos] } \\
\left(\mathrm{LD}^{18}\right)\end{array}$ & & $\begin{array}{l}\text { Nahi ere nahi du beldur eta aztora } \\
\text { daitezen. [Querer, eso es lo que } \\
\text { quiere, que tengan miedo y que se } \\
\text { inquieten] }\left(\mathrm{LD}-\mathrm{eu}^{19}\right)\end{array}$ \\
\hline
\end{tabular}

Los dos primeros ejemplos corresponden a la no-traducción de la partícula. Los dos son traducciones indirectas, pero en el primer ejemplo la versión puente española no recoge el matiz de la partícula, y, por tanto, tampoco lo hace la versión en euskera. En el segundo ejemplo, sin embargo, aunque la versión puente española recoge el matiz de la partícula, la versión en euskera tampoco lo traduce. De todos modos, hemos encontrado casos en los que la versión meta en euskera sí capta el matiz de la partícula que aparecía ya traducida en la versión puente española (ejemplo número 4). Esto nos lleva a la reflexión de que la intermediación de la versión puente conlleva las siguientes tres principales posibilidades: una, que la versión puente refleje el matiz 
de la partícula pero la versión meta no; dos, que la versión puente traslade el matiz de la partícula y la versión puente lo haga también; y tres, que la versión puente no recoja el matiz de la partícula y, por ende, tampoco la versión meta. Cabría una cuarta posibilidad, improbable pero no del todo descartable: que la versión puente no refleje la partícula existente en el texto original alemán pero sí se constate una equivalencia idiomática en el texto meta en euskera, ya sea porque el traductor haya recurrido al texto alemán o porque el idioma meta brinda la oportunidad de hacerlo y el traductor vasco haya realizado por casualidad una traducción compensatoria.

En cuanto a los ejemplos 3, 4 y 5, se reflejan algunas de las estrategias utilizadas para recoger el matiz de la partícula. En el tercer ejemplo el traductor ha utilizado una construcción causal (en euskera, una de las opciones para hacer frases causales es la de añadir -eta [y] al final); en el cuarto ejemplo se ha utilizado una frase negativa (al igual de lo que hace la versión puente); y en el quinto ejemplo, el traductor ha utilizado el recurso de repetir el verbo para enfatizarlo (en este caso, repite el verbo nahi [querer]). Ciertamente, estos recursos logran recoger el matiz de la partícula alemana del texto original. De todos modos, harían falta más estudios para analizar sistemáticamente su uso como estrategias traductoras. Siendo éste un estudio descriptivo, nos limitamos a presentar la posibilidad de estos recursos.

Recuperando la información que teníamos sobre los tipos de ja, hemos observado si existe alguna relación entre el tipo de ja (de conocimiento, asombro o imperativo) y la proporción de traducción de la partícula. Al analizar los casos seleccionados, concluimos que el matiz de la partícula se reflejaba más en los ja de asombro y los ja imperativos. Así pues, hemos cruzado ambas informaciones y los resultados los hemos representado en el siguiente gráfico:

\section{FIGURA 2}

Porcentajes de las estrategias de traducción según el tipo de ja

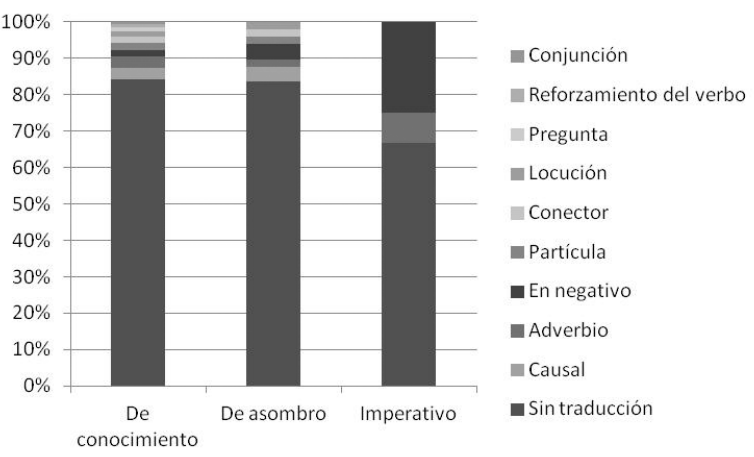

Según nos muestra el gráfico, la partícula ja de asombro se ha traducido en más ocasiones que la de conocimiento ( $3 \%$ más), pero la gran diferencia la marca la partícula ja imperativa, ya que se ha traducido un $18 \%$ más que la de conocimiento, en la mayoría de los casos a través de una frase negativa (la frase 4 de la anterior tabla sería un ejemplo de una frase negativa). En vista de los resultados, se podría concluir que los ja imperativos son los que ejercen una función ilocutiva más notoria o que los traductores reconocen como tales y, por tanto, se mantienen más que los demás.

A continuación, vamos a analizar si también existe alguna diferencia entre traducciones directas e indirectas a la hora de traducir las partículas. He aquí los gráficos: 
FIGURA 3

Estrategias de traducción de ja en traducciones directas (izquierda) y traducciones indirectas (derecha)
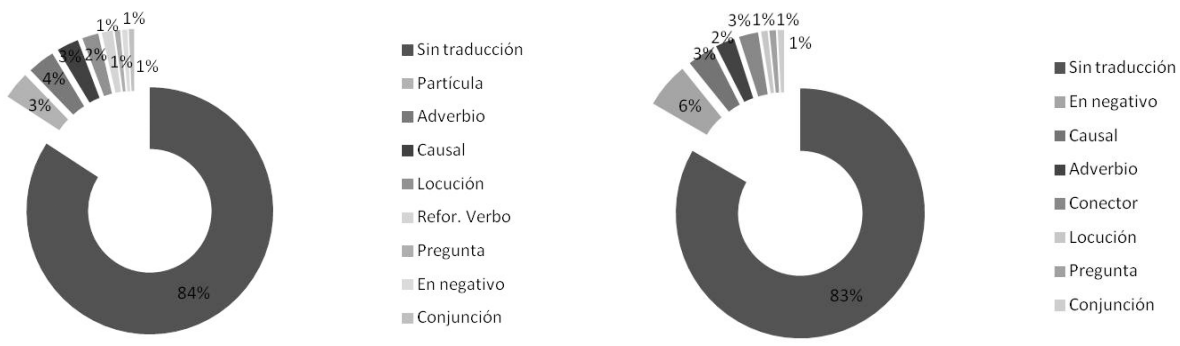

La traducción de la partícula ja se ha reflejado el 1\% más en las traducciones indirectas que en las directas. Aunque las cifras son muy similares, parece que la intermediación de un texto puente haya podido propiciar que la partícula se refleje en mayor medida en los textos meta. Además, cabe destacar que ambos resultados numéricos no se pueden valorar según la misma escala: las traducciones indirectas se realizan a partir del filtro que suponen las versiones intermediarias; con lo cual se podría afirmar que el $17 \%$ de traducción de la partícula de las versiones indirectas podría equivaler más que el 16\% de traducción de las traducciones directas (más que el $1 \%$ de diferencia que ya se constata). Tal y como habíamos adelantado, si la versión puente de las traducciones indirectas no traslada la partícula de forma suficientemente clara, lo más probable es que este matiz se pierda en la versión en euskera, ya que esta última versión desconoce que en la versión original alemana haya ninguna partícula modal. Según nuestro análisis, los casos en los que la versión meta en euskera no refleja la traducción del matiz que la versión puente sí refleja son el 10\%.

Según los datos observados hasta ahora, la estandarización en cuanto a la función ilocutiva que desempeñan las partículas modales es clara, tanto en traducciones directas como en indirectas. En lo que se refiere a la interferencia, hemos observado con especial atención las posibles interferencias de una versión intermediaria en las traducciones directas. Tal y como se ha comprobado en más de un estudio del grupo de investigación TRALIMA/ITZULIK (Sanz 2013; Barambones, Manterola et al. 2015), es posible percibir interferencias de un texto puente en las traducciones supuestamente directas realizadas al euskera. En el caso concreto de las traducciones de la partícula ja, el siguiente ejemplo nos llevó a consultar la versión en español y a concluir que el traductor se guió por dicha versión.

CUADRO 2

Interferencia de una versión puente en una traducción supuestamente directa

\begin{tabular}{|l|l|l|}
\hline \multicolumn{1}{|c|}{ Texto original } & \multicolumn{1}{|c|}{ Versión en español } & \multicolumn{1}{c|}{ Texto meta } \\
\hline $\begin{array}{l}\text { Natürlich, Almut und Reinhard } \\
\text { waren ja um diese Zeit in der } \\
\begin{array}{l}\text { Schule. [Naturalmente, aquella } \\
\text { hora Almut y Reinhard } \\
\text { estaban, cómo no, en la } \\
\text { escuela] }\end{array}\end{array}$ & $\begin{array}{l}\text { Naturalmente, Almut y } \\
\text { Reinhard estarían en la escuela } \\
\left(\mathrm{WO}^{20}\right)\end{array}$ & $\begin{array}{l}\text { Jakina, Almut eta Reinhard } \\
\text { eskolan zeuden, nonbait. [Por } \\
\text { supuesto, Almut y Reinhard } \\
\text { estaban, según parece, en la } \\
\text { escuela] }\end{array}$ \\
\hline
\end{tabular}


En el trascurso del análisis textual nos sorprendió que el traductor de la versión meta incluyese el adverbio nonbait [parece ser], ya que la partícula ja aporta a la frase original cierto matiz de conocimiento y seguridad de que los citados personajes estaban en la escuela. Decidimos consultar la versión española, ya que, además, en el análisis macrotextual de la traducción de esta obra en concreto (die Wolke) pudimos observar que le traducción meta en euskera no llevaba el prólogo de la obra original, al igual que no lo llevaba la versión en español (publicada con anterioridad a la versión en euskera). Para este ejemplo en concreto, volvimos a consultar la traducción al español y pudimos deducir que el traductor al euskera había podido guiarse por dicha versión, pues la utilización de nonbait derivaría del verbo español en sentido hipotético estarían. Con este ejemplo nuestro objetivo no es sancionar el hecho de que los traductores utilicen en mayor o menor medida textos intermediarios. Sin embargo, este tipo de ejemplo reafirma el carácter «supuesto» de las traducciones directas.

\subsection{Resultados del análisis de las partículas eben/halt}

En el caso de la partícula eben, también tuvimos que hacer una selección, ya que eben, además de partícula modal, existe como partícula de respuesta: $\underline{\mathrm{Na} \text {, eben! [jexacta- }}$ mente!], como partícula de graduación: Hier eben habe ich ihn getroffen [me lo he encontrado justamente aquí] o como adjetivo: Eine ebene Fläche [una superficie plana]. Así, de entre los 125 resultados obtenidos en la búsqueda, los correspondientes a esta partícula modal eran 93. En cuanto a las funciones que adquiere como partícula modal (Cárdenes 1997: 163), en oraciones enunciativas expresa la resignación del hablante: Es gibt eben keine geraden Schweineschwänzchen [En fin, no existe ninguna cola de cerdo que sea recta]. En oraciones imperativas, en cambio, el hablante busca el compromiso o la aceptación del interlocutor: Das müssen die eben zahlen! [¡Pues tendrán que pagar!]. En cuanto a halt, tras prescindir del homónimo del verbo halten [parar] en forma imperativa, hemos obtenido 38 resultados. Se la considera sinónimo de eben, por lo que las funciones que cumple dentro de la oración son idénticas a las de eben.

En lo que se refiere a las estrategias de traducción utilizadas, y tal y como ocurría con la partícula ja, la no-traducción ha sido la más utilizada para la partícula eben. La cifra es además muy parecida (83\%), pues recordemos que en el caso de ja la proporción de no-traducción era del $84 \%$. Por tanto, se confirma la estandarización en cuanto a lo que se refiere a la función que las partículas modales cumplen en el texto original.

FIGURA 4

Porcentajes de las estrategias de traducción de la partícula eben

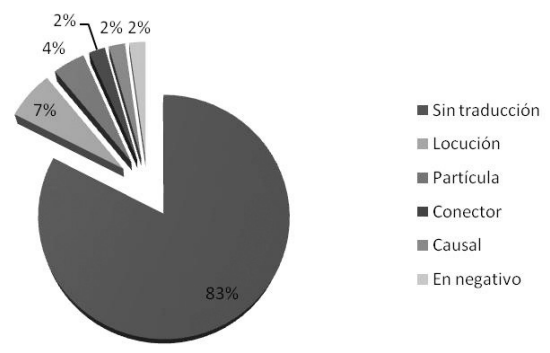


En cuanto a la partícula halt, hemos identificado la no-traducción 32 veces (84\% de los casos) y se ha recogido la traducción 6 veces (16\% de los casos). Por tanto, la traducción de la partícula halt también responde a la tendencia de no-traducción que hemos estado observando hasta ahora. A continuación, dos ejemplos en los que se traduce el matiz de la partícula eben. Los dos son traducciones directas.

CUADRO 3

Estrategias de traducción de la partícula eben

\begin{tabular}{|l|l|}
\hline \multicolumn{1}{|c|}{ Texto original } & \multicolumn{1}{|c|}{ Texto meta } \\
\hline $\begin{array}{l}\text { 1. „Es gibt eben keine geraden } \\
\text { Schweineschwänzchen” [Es que no } \\
\text { existe ninguna cola de cerdo que sea } \\
\text { recta] }\left(\mathrm{FR}^{23}\right)\end{array}$ & $\begin{array}{l}\text {-Tira, ez dago txerri-buztan zuzenik. } \\
\text { [En fin, no existe ninguna cola de cerdo } \\
\text { recta] }\left(\mathrm{FR}_{\left.-1 u^{24}\right)}\right.\end{array}$ \\
\hline $\begin{array}{l}\text { 2. „Das müssen die eben zahlen!” [iPues } \\
\text { eso lo tienen que pagar, y punto!] (LD; } \\
\text { ver nota 18) }\end{array}$ & $\begin{array}{l}\text { - } \text { Ba, ordaindu egin beharko dute! } \\
\text { [Pues, tendrán que pagarlo!] (LD-eu; } \\
\text { ver nota 19) }\end{array}$ \\
\hline
\end{tabular}

En el primer ejemplo se ha utilizado la expresión tira [en fin], que sugiere resignación. En el segundo ejemplo, en cambio, se ha utilizado la partícula ba(da) [pues] que también expresa resignación. Tal y como ocurría con la partícula ja, encontramos recursos que pueden servir como estrategias de traducción de la partícula eben $\underline{\text { halt, }}$, pero harían falta más estudios para verificar la posibilidad de utilizar estos recursos sistemáticamente. En cuanto a la posible diferencia entre traducciones directas e indirectas, he aquí los dos gráficos que se refieren a la partícula eben ${ }^{25}$ :

FIGURA 5

Porcentajes de traducción de la partícula eben en traducciones directas (izquierda) $\mathrm{y}$ traducciones indirectas (derecha)
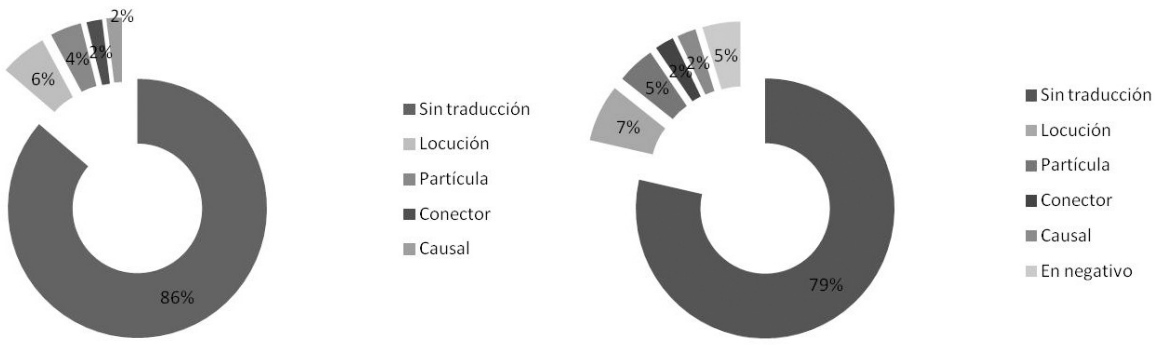

Aunque esta vez la diferencia tampoco es muy grande, en las traducciones indirectas se ha traducido eben un 7\% más que en las traducciones directas. Recordemos que la diferencia en el caso de ja era del 1\%, también a favor de las traducciones indirectas. Estó indicaría que, según el estudio realizado en nuestro corpus, el texto intermediario sí afecta a la traducción de las partículas modales alemanas analizadas. En principio, la existencia de un texto puente podría llevarnos a concluir lo contrario, ya que en las traducciones indirectas el proceso de traducción se desarrolla en dos pasos y la posibilidad de perder matices como los que aportan las partículas modales debería ser mayor. Sin embargo, la estrategia utilizada por la versión puente condiciona siempre el resultado final del texto meta y, en este caso, este condicionamiento 
aporta un índice de traducción de las partículas mayor que lo que aportan las traducciones directas.

Por otro lado, en el trascurso del análisis de las estrategias de traducción, también hemos detectado algún que otro caso de interferencia, resultado del uso de un texto intermediario. En el siguiente ejemplo, la interferencia no se refiere exactamente a la partícula, sino al adjetivo, pero la detectamos durante el análisis de la traducción de la partícula.

CUADRO 4

Interferencia de un texto puente en una traducción supuestamente directa

\begin{tabular}{|c|c|c|}
\hline Texto original & Versión en español & Texto meta \\
\hline $\begin{array}{l}\text { „Ich kann's eben nicht anders } \\
\text { ausdrücken”, sagte Tünnes } \\
\text { verlegen. ["Pues es que no lo } \\
\text { puedo expresar de otra } \\
\text { manera", dijo Tünnes } \\
\frac{\text { avergonzado] (WO; ver nota }}{20 \text { ) }}\end{array}$ & $\begin{array}{l}\text {-No lo sé decir de otra manera } \\
\text {-dijo Tünnes desconcertado. } \\
\text { (WOes; ver nota 21) }\end{array}$ & $\begin{array}{l}\text {-Ez dakit beste era batera } \\
\text { esaten -esan zuen Tünnesek } \\
\text { aztoraturik. [-No lo sé decir de } \\
\text { otra manera -dijo Tünnes } \\
\text { inquieto.] (WO-eu; ver nota 22) }\end{array}$ \\
\hline
\end{tabular}

El traductor del texto meta traduce verlegen [avergonzado] como aztoraturik [nervioso, inquieto], lo que había despertado nuestras sospechas. Además, en la traducción del artículo ja habíamos detectado un caso similar relativo a este mismo libro (die Wolke). Tras consultar la versión en español, se podría pensar que la traducción al euskera de aztoraturik surge de la traducción española desconcertado.

\section{Conclusiones}

El objetivo de este artículo era analizar la traducción al euskera de las partículas modales alemanas, en cuanto a que son elementos lingüísticos pertenecientes al discurso oral. Tal y como hemos podido comprobar, la estrategia de traducción más utilizada ha sido la no-traducción de la partícula. Por tanto, la estandarización en cuanto al matiz que aportan las partículas modales alemanas es clara. Cabe recordar que las partículas modales son muy habituales en diálogos y en registros no formales, y puede que esta comparativa signifique que en euskera la insistencia de elementos lingüísticos de carácter ilocutivo no sea una convención en el mismo nivel que en alemán, o puede que cada lengua compense dicha función de alguna otra manera en el texto. De todos modos, hemos podido comprobar que sí hay medios para poder mantener el matiz que la partícula modal aporta al texto original: en el caso de la partícula ja, la construcción causal o la repetición del verbo, si se trata de un ja de conocimiento, o la frase en negativo si se trata de un ja imperativo; en el caso de eben, hemos visto la posibilidad de utilizar la interjección tira [bueno] o la partícula ba(da) [pues]. De todos modos, y según los resultados de nuestro análisis, el uso de estos recursos parece ser limitado. Prüfer comenta que dos de las obras de su corpus que se publicaron en los años 1990 tienen traducidas el 50\% de las partículas, mientras que dicho porcentaje cae al 20-25\% en las obras publicadas en la década de los años 1980. Según sostiene Prüfer, el aumento de dicho porcentaje es gracias al logro de la investigación de partículas y la enseñanza de idiomas (Prüfer 1995: 241). Puede que el desconocimiento pragmático de la lengua alemana haya sido una de las causas por 
la que los traductores vascos no hayan recogido en mayor medida el matiz de las partículas modales, y tal vez el posible desarrollo de materiales y manuales en esta combinación lingüística fomente el uso de algunos de los recursos aquí mencionados como estrategias de traducción de las partículas. De todos modos, harían falta más estudios sobre el tema para poder analizar sistemáticamente y con más profundidad el uso de los recursos que hemos observado en nuestro estudio. Nuestro análisis ha sido un primer paso descriptivo, pero si futuros estudios confirmasen algunos de los recursos observados en nuestro trabajo, se podría adoptar una actitud más prescriptivista, pudiéndose dar recomendaciones concretas para el trasvase al euskera de las partículas modales. Al mismo tiempo, sería conveniente realizar no sólo estudios sincrónicos sino también diacrónicos que permitan analizar la evolución de la traducción de las partículas modales al euskera en la literatura infantil y juvenil. Además, sería interesante compilar corpus específicos (sólo de literatura infantil o sólo de literatura juvenil), a fin de realizar análisis más concretos. Tal y como hemos mencionado, este estudio ha sido un primer análisis de carácter descriptivo. A fin de poder aplicar la descripción a la práctica diaria de los traductores, lo ideal sería analizar sistemáticamente algunos de los resultados aquí obtenidos.

La comparación entre las traducciones directas e indirectas ha dejado ver que, aunque por un porcentaje pequeño, las traducciones indirectas recogen un poco más el matiz de la partícula. Relacionamos este hecho con la interferencia que el texto intermediario pueda ejercer o no en el texto meta: en principio, en una traducción indirecta el traductor del texto meta desconoce la existencia de ninguna partícula y sólo podrá recoger el matiz original de aquella si el traductor del texto puente lo recoge claramente; en este caso, parece que las estrategias adoptadas por el texto puente han propiciado un mayor índice de traducción de las partículas. Por tanto, hemos detectado la estandarización de las partículas modales alemanas, pero también hemos identificado que la traducción indirecta disminuye en cierta medida este hecho. Además de la estandarización, cabe destacar que hemos detectado algunos casos de interferencia del texto intermediario en las traducciones supuestamente directas. Este hecho no es extraño en el caso de la lengua vasca, pues convive con el español y, en la mayoría de los casos, las obras se publican en español antes que en euskera. Asimismo, aunque existe un pequeño diccionario alemán-euskera-alemán, la mayoría de las herramientas y materiales se refieren a la combinación lingüística alemán-español. Al mismo tiempo, no podemos olvidar la interferencia que acompaña al traductor en sí. Todo ello hace que la interferencia de un texto/una lengua intermediaria sea casi inevitable en el caso de lenguas minorizadas como el euskera. Creemos, por tanto, que el concepto de interferencia que Toury (2012) utiliza para proponer la ley de traducción se podría aplicar tanto a las traducciones indirectas como a las supuestamente directas. Además, sostenemos que la interferencia es distinta en el caso de las traducciones realizadas a una lengua minorizada o diglósica. No es lo mismo traducir del alemán al inglés que del alemán al euskera, ya que en el segundo caso la interferencia es ocasionada por la lengua hegemónica, tanto en traducciones indirectas como en las supuestamente directas. Incluso se podría analizar si también encontramos esta interferencia en textos originales en la lengua minorizada.

A modo de conclusión, pensamos que cuestiones como la estandarización del discurso oral y la interferencia de las lenguas mayoritarias que funcionan como 
puente se verán reflejadas en mayor medida en lenguas minorizadas como el euskera. Más estudios sobre la traducción de elementos del discurso oral contribuirían a profundizar en la reflexión sobre la oralidad fingida de lenguas minorizadas como la lengua vasca y a observar el efecto de otras lenguas sobre ella.

\section{NOTAS}

1. Utilizamos el término «disfemización» para designar lo contrario de «eufemización», es decir para los casos en los que se neutraliza el tono o registro del texto original. Se trata del término propuesto también por Martí (2006: 70) para describir la traducción de subtítulos y doblaje de productos audiovisuales del inglés al español.

2. Entendemos por «lengua minorizada» aquella cuyo uso está restringido por motivos políticos y sociales o como consecuencia de una relación de subordinación.

3. Aplicamos el concepto «assumed» que utiliza Toury (2012: 35) para enunciar el concepto de «supuesta traducción» a las traducciones directas e indirectas. Para esclarecer este dato provisional hace falta un análisis microtextual.

4. TRALIMA/ITZULIK - Traducción, Literatura y Medios Audiovisuales, grupo de investigación consolidado: GIC 12_197 IT728-13, UFI 11_06 UPV-EHU, <http://www.ehu.es/tralima/inicio_eus. php>.

5. TRACE - Traducciones censuradas, proyecto de investigación, <http://www.ehu.es/trace/colectivo. html>.

6. Como aclaración del número total del que se compone el corpus digital alineado: 19 textos originales con sus traducciones al euskera (traducciones directas): $19 \times 2=38$ textos. 14 textos originales con sus traducciones al español y al euskera (traducciones indirectas): $14 \times 3=42$ textos. En total, $38+42=80$ textos alineados.

7. Preussler, Otfried (1966): Das kleine Gespenst. Stuttgart: Thienemanns Verlag.

8. Preussler, Otfried (1969): El pequeño fantasma (Traducido por Carmen Maluenda). Barcelona: Bruguera.

9. Preussler, Otfried (1984): Fantasmatxoa (Traducido por Xabier GartZia). Donostia: ELKAR.

10. Press, Hans Jürgen (1965): Die Abenteuer der schwarzen Hand. Dillingen: Otto Maier Verlag Ravensburg.

11. Press, Hans Jürgen (1981): Aventuras de "la mano negra" (Traducido por José SÁnCHez). Madrid: Espasa-Calpe.

12. Press, Hans Jürgen (1984): Eskubeltz taldearen abenturak (Traducido por Pilar IparRagirRe). Donostia: ELKAR.

13. Grimm, Brüder (1812-1815): Kinder und Hausmärchen. Projekt Gutengerg-DE. Spiegel Online Kultur. Consultado el 13 de noviembre, <http://gutenberg.spiegel.de/buch/6248/1>.

14. Grimm, Brüder (1999): Grimm anaien ipuinak (Traducido por Genaro Gomez). Iruña: Pamiela.

15. KäSTnER, Erich (1929). Emil und die Detektiven. Zürich: Atrium Verlag.

16. Kästner, Erich (1967): Emilio y los detectives (Traducido por José Fernández). Barcelona: Juventud.

17. KäStNer, Erich (1991): Emilio eta detektibeak (Traducido por Tomás Sarasola). Donostia: ELKAR.

18. Härtling, Peter (1993): Lena auf dem Dach. Weinheim: Beltz \& Gelberg.

19. Hërtling, Peter (1994): Lena teilatuan (Traducido por Pello Zabaleta). Donostia: ElKAR.

20. Pausewang, Gudrun (1987): Die Wolke. Dillingen: Ravensburger Buchverlag.

21. Pausewang, Gudrun (1988): La nube (Traducido por Lorenzo Rodríguez). Salamanca: Loguez.

22. Pausewang, Gudrun (1991): Hodeia (Traducido por Xabier Mendiguren). Donostia: ElKAR.

23. Härtling, Peter (1989): Fränze. Weinheim: Beltz \& Gelberg.

24. Härtling, Peter (1992): Franze (Traducido por Xabier Mendiguren). Donostia: ElKAR.

25. Los resultados de halt no los hemos representado gráficamente, pues se trataba de un número reducido. 


\section{REFERENCIAS BIBLIOGRÁFICAS}

AÑoRgA, Pello (2011): Haur-literatura eta ahozkotasuna [Literatura infantil y oralidad]. Behinola. 24:21-29.

BARAmbones, Josu (2010): La traducción audiovisual en ETB-1: Estudio descriptivo de la programación infantil y juvenil. Tesis de doctorado. Vitoria-Gasteiz: Universidad del País Vasco/ Euskal Herriko Unibertsitatea.

Barambones, Josu (2012): Lenguas minoritarias y traducción. La traducción audiovisual en euskera. Castellón de la Plana: Universitat Jaume I.

Barambones, Josu, Manterola, Elizabete, SAnZ, Zuriñe, et al. (2015): Itzulpen ikasketak eta euskara. Zenbait ekarpen Itzulpen Ikasketa Deskribatzaileei, eta haratago [Los Estudios de Traducción y el euskera. Algunas aportaciones a los Estudios Descriptivos de Traducción y más allá]. In: Traducimos desde el Sur Actas del VI Congreso Internacional de la Asociación Ibérica de Estudios de Traducción e Interpretación. Las Palmas de Gran Canaria: ULPGC.

BeErbom, Christiane (1992): Modalpartikeln als Übersetzungsproblem. Eine kontrastive Studie zum Sprachenpaar Deutsch-Spanisch. Fráncfort: Peter Lang.

Ben-Ari, Nitsa (1992): Didactic and Pedagogic Tendencies in the Norms Dictating the Translation of Children's Literature: The Case of Postwar German-Hebrew Translations. Poetics Today. 13(1):221-231.

Bendel, Christiane (2006): Baskische Grammatik. Hamburgo: Helmut Buske Verlag.

Brumme, Jenny y Espunya, Anna (2012): The Translation of Fictive Dialogue. Amsterdam/Nueva York: Rodopi.

CÁrdenes, José (1997): Aber, denn, doch, eben und ihre spanischen Entsprechungen. Eine funktional-pragmatische Studie zur Übersetzung deutscher Partikeln. Münster/Nueva York/ Múnich/Berlín: Waxmann.

ESNAOLA, Imanol (1997): Gazte euskaldunen arteko hizkera hemen eta orain [El lenguaje juvenil vasco aquí y hoy]. Bilbao: Udako Euskal Unibertsitatea.

Etxaniz, Xabier (2011): Sarrera. Egungo euskal haur eta gazte literatura [Introducción. La literatura infantil y juvenil vasca actual.]. In: Xabier ETXAniz y Manu Lopez eds. Egungo euskal haur eta gazte literaturaren historia [Historia de la literatura infantil y juvenil vasca actual]. Bilbao: Servicio editorial de la Universidad del País Vasco.

FERrer, Hang (1999): Las partículas modales alemanas en el modo interrogativo y sus equivalencias en español como criterio para una taxonomia de preguntas. Valencia: Universitat de València.

Fischer, Martin B. y Wirf Naro, Maria (2012): Translating Fictional Dialogue for Children and Young People. Berlín: Frank \& Timme.

HeLbig, Gerhard (1988): Lexikon deutscher Sprache. Leipzig: VEB.

Lambert, José y van Gorp, Hendrik (1985). On describing translations. In: Theo Hermans ed. The Manipulation of Literature: Studies in Literary Translation. Londres/Sídney: Croom Helm, 42-53.

MARTí, José Luis (2006): Estudios empírico y descriptivo del método de traducción para doblaje y subtitulación. Castellón de la Plana: Universitat Jaume I.

Neuland, Eva (2007): Subkulturelle Sprachstile Jugendlicher heute. Tendenzen der Substandardisierung in der deutschen Gegenwartssprache. In: Eva Neuland, ed. Jugendsprache, Jugendliteratur, Jugendkultur. Fráncfort: Peter Lang, 131-149.

PIĘTA, Hanna (2012): Patterns in (in)directness. An exploratory case study in the external history of Portuguese translations of Polish literature (1855-2010). Target. 24(2):310-337.

PRÜFER, Irene (1995): La traducción de las partículas modales del alemán al español y al inglés. Fráncfort: Peter Lang.

Ringmar, Martin (2007): Roundabout Routes: Some remarks on indirect translations. Selected Papers of the CETRA Research Seminar in Translation Studies 2006. Consultado el 15 de diciembre de 2015, <http://www2.arts.kuleuven.be/info/bestanden-div/RINGMAR.pdf>. 
SANZ, Zuriñe (2013): Korpusbasierte Übersetzungsanalyse von Hand-Somatismen (deutschbaskisch). In: Melanija Fabčič, Sabine Fiedler y Joanna Szerszunowicz eds. Phraseologie im interlingualen und interkulturellen Kontakt. Maribor: Zora, 317-330.

Shavit, Zohar (1986): Poetics of Children's Literature. Athens, EUA/Londres: University of Georgia Press.

Toury, Gideon (2012): Descriptive Translation Studies - and beyond. Revised edition. Amsterdam/Filadelfia: John Benjamins.

URIBARRI, Ibon (2011): Dialectics of Opposition and Construction: Translation in the Basque Country. In: Dimitris Asimakoulas y Margaret Rogers, eds. Translation and Opposition. Brístol: Multilingual Matters, 247-264.

Venuti, Lawrence (1998): Introduction. Translation and Minority. The Translator. 4(2):135-144.

WALtereit, Richard (2006): Zur Pragmatik und historischen Semantik von Modalpartikeln und ihren funktionalen Äquivalenten in romanischen Sprachen. Kempten: Max Niemeyer Verlag.

ZubillagA, Naroa (2009): Alemanetik euskaratutako haur eta gazte literatura edo amaigabeko istorioa [Traducción de la literatura infantil y juvenil alemana al euskera o la historia interminable]. Senez. 37:231-241.

Zubillaga, Naroa (2012): A corpus based descriptive study of German children's literature translated into Basque: Preliminary results. In: Martin Fischer y Maria Wirf Naro, eds. Translating Fictional Dialogue for Children and Young People. Berlín: Frank \& Timme, 81-99.

Zubillaga, Naroa (2013): Alemanetik euskaratutako haur-eta gazte-literatura: zuzeneko nahiz zeharkako itzulpenen azterketa corpus baten bidez [Traducción de literatura infantil y juvenil al euskera: comparación de traducciones directas e indirectas a través de un corpus]. Tesis de doctorado. Vitoria-Gasteiz: Universidad del País Vasco/Euskal Herriko Unibertsitatea. Consultado el 15 de diciembre de 2015. < https://addi.ehu.es/bitstream/10810/1243 1/1/8670ZubillagaEU.pdf $>$.

Zubillaga, Naroa, SAnZ, Zuriñe y URIBARri, Ibon (2015): Building a trilingual parallel corpus to analyse literary translations from German into Basque. In: Claudio Fantinuoli y Federico Zanettin, eds. New Directions in Corpus-based Translation Studies. Berlín: Language Science Press, 71-92. 